\title{
Long-term safety and efficacy of pegvaliase for the treatment of phenylketonuria in adults: combined phase 2 outcomes through PAL-003 extension study
}

Nicola Longo ${ }^{1 *}$, Roberto Zori ${ }^{2}$, Melissa P. Wasserstein ${ }^{3}$, Jerry Vockley ${ }^{4}$, Barbara K. Burton ${ }^{5}$, Celeste Decker ${ }^{6}$, Mingjin Li ${ }^{6}$, Kelly Lau ${ }^{6}$, Joy Jiang ${ }^{6}$, Kevin Larimore ${ }^{6}$ and Janet A. Thomas ${ }^{7}$

\begin{abstract}
Background: Deficiency of phenylalanine hydroxylase causes phenylketonuria (PKU) with elevated phenylalanine (Phe) levels and associated neuropsychiatric and neurocognitive symptoms. Pegvaliase (PEGylated phenylalanine ammonia lyase) is an investigational agent to lower plasma Phe in adults with PKU. This study aimed to characterize the long-term efficacy, safety, and immunogenicity of pegvaliase in adults with PKU.

Methods: PAL-003 is an ongoing, open-label, long-term extension study of the pegvaliase dose-finding parent phase 2 studies. Participants continued the dose of pegvaliase from one of three parent studies, with dose adjustments to achieve a plasma Phe concentration between 60 and $600 \mu \mathrm{mol} / \mathrm{L}$.

Results: Mean (standard deviation [SD]) plasma Phe at treatment-naïve baseline for 80 participants in the parent studies was 1302.4 (351.5) $\mu \mathrm{mol} / \mathrm{L}$. In the 68 participants who entered the extension study, plasma Phe decreased 58.9 (39)\% from baseline, to 541.6 (515.5) $\mu \mathrm{mol} / \mathrm{L}$ at Week 48 of treatment. Plasma Phe concentrations $\leq 120 \mu \mathrm{mol} / \mathrm{L}$, $\leq 360 \mu \mathrm{mol} / \mathrm{L}$, and $\leq 600 \mu \mathrm{mol} / \mathrm{L}$ were achieved by $78.7,80.0$, and $82.5 \%$ of participants, respectively. Mean (SD) protein intake at baseline was 69.4 (40.4) $\mathrm{g} /$ day (similar to the recommended intake for the unaffected population) and remained stable throughout the study. All participants experienced adverse events (AEs), which were limited to mild or moderate severity in most (88.8\%); the most common AEs were injection-site reaction (72.5\%), injection-site erythema (67.5\%), headache (67.5\%), and arthralgia (65.0\%). The AE rate decreased from 58.3 events per person-year in the parent studies to 18.6 events per person-year in the extension study.
\end{abstract}

Conclusions: Pegvaliase treatment in adults with PKU produced meaningful and persistent reductions in mean plasma Phe concentration with a manageable safety profile for most subjects that continued with long-term treatment.

Trial registration: ClinicalTrials.gov, NCT00924703. Registered June 18, 2009, https://clinicaltrials.gov/ct2/show/ NCT00924703

Keywords: Phenylketonuria, PKU, Recombinant Anabaena variabilis PEGylated phenylalanine ammonia lyase, Pegvaliase

\footnotetext{
* Correspondence: nicola.longo@hsc.utah.edu

1 Department of Pediatrics, Division of Medical Genetics, University of Utah,

295 Chipeta Way, Salt Lake City, UT 84108, USA

Full list of author information is available at the end of the article
}

(c) The Author(s). 2018 Open Access This article is distributed under the terms of the Creative Commons Attribution 4.0 International License (http://creativecommons.org/licenses/by/4.0/), which permits unrestricted use, distribution, and reproduction in any medium, provided you give appropriate credit to the original author(s) and the source, provide a link to the Creative Commons license, and indicate if changes were made. The Creative Commons Public Domain Dedication waiver (http://creativecommons.org/publicdomain/zero/1.0/) applies to the data made available in this article, unless otherwise stated. 


\section{Background}

Phenylketonuria (PKU; OMIM 261600) is an autosomal recessive disorder with an incidence in the United States of approximately 1 in 16,500 newborns [1]. PKU is caused by a deficiency of phenylalanine hydroxylase, the enzyme that converts phenylalanine (Phe) to tyrosine, resulting in an accumulation of Phe in the blood and brain [2].

In some patients, chronically elevated Phe levels can lead to a range of developmental, neurocognitive, and neuropsychiatric comorbidities [3]. Despite advances in treatment, $20-65 \%$ of adults with PKU fail to achieve metabolic control. This stems from a number of factors, including issues with adherence to treatment, access to care, and the limited efficacy of available therapeutic interventions [4-8].

Guidelines from the American College of Medical Genetics and Genomics (ACMG) recommend lifelong treatment to maintain plasma Phe concentration between $120 \mu \mathrm{mol} / \mathrm{L}$ and $360 \mu \mathrm{mol} / \mathrm{L}$ to minimize the adverse impact of elevated Phe on the brain [2]. Treatment goals related to plasma Phe are individualized based on the impact of high Phe concentrations on patients [9]. Individuals with PKU are counseled to lower plasma Phe with a diet severely restricted in proteins and supplemented with low-Phe or Phe-free, amino acid-modified medical foods or special low-protein foods, with or without sapropterin dihydrochloride (KUVAN ${ }^{\circledR}$, BioMarin Pharmaceutical Inc., Novato, CA), which is indicated for the treatment of PKU in combination with diet $[2,8]$. Pegvaliase Palynziq $^{\mathrm{TM}}$, BioMarin Pharmaceutical Inc.) is a Phe-metabolizing enzyme recently approved in the US to reduce plasma Phe concentrations in adults with PKU who have plasma Phe concentrations $>600 \mu \mathrm{mol} / \mathrm{L}$ [10].

Pegvaliase is a recombinant Anabaena variabilis phenylalanine ammonia lyase (PAL) enzyme conjugated to polyethylene glycol (PEG) to optimize pharmacodynamic stability and reduce immune response to PAL [11]. PAL converts Phe to ammonia and trans-cinnamic acid, which are readily metabolized by the liver and excreted in the urine [10-12].

The phase 1 study PAL-001, a dose-escalation study of pegvaliase in adults with PKU, showed that a single dose of $0.1 \mathrm{mg} / \mathrm{kg}$ reduced plasma Phe levels from baseline and maintained the reduction for up to 1 week [11]. The first phase 2 study, PAL-002, a dose-finding study of pegvaliase, demonstrated that once-weekly dosing at $0.001 \mathrm{mg} /$ $\mathrm{kg}$ to $0.1 \mathrm{mg} / \mathrm{kg}$ for 16 weeks was generally well tolerated, but did not result in significant plasma Phe reductions. In the subsequent phase 2 study PAL-004, pegvaliase at $0.06 \mathrm{mg} / \mathrm{kg}$ to $0.4 \mathrm{mg} / \mathrm{kg}$ for 5 days/week caused immediate and substantial plasma Phe reductions. However, many participants had to reduce or interrupt pegvaliase due to hypersensitivity events, and plasma Phe reduction was not sustained at the end of 13 weeks of treatment [13]. In the third phase 2 study, 165-205, pegvaliase was started at a low dose (2.5 mg/week) that was gradually increased (to a maximum of $375 \mathrm{mg} /$ week) to minimize hypersensitivity events. This pegvaliase dosing regimen with an induction, titration, and maintenance period led to substantial reductions in mean plasma Phe concentration during the 24-week study [14].

Here we report on the longest duration of pegvaliase treatment to date. Long-term efficacy, safety, and immunogenicity of pegvaliase was assessed in participants who initiated treatment in PAL-002 (NCT00925054) [13], PAL-004 (NCT01212744) [13], or 165-205 (NCT01560286) [14] and then continued on in the PAL-003 extension study reported here.

In addition to the studies described above, two phase 3 clinical trials of pegvaliase, which included a double-blind, randomized withdrawal trial [15] and an open-label, long-term extension study [16], were recently published. These phase 3 studies evaluated the efficacy and safety of pegvaliase dosed at 5 to $60 \mathrm{mg} /$ day.

\section{Methods \\ Study design}

PAL-003 is an open-label, multicenter, long-term phase 2 extension study of pegvaliase treatment in adults with PKU who completed a prior dose-finding parent phase 2 study. The primary objective of this study was to evaluate the long-term effect of pegvaliase on plasma Phe concentration; secondary objectives were to evaluate safety and immune response; and an exploratory objective was to assess the relationship between dietary protein intake and changes in plasma Phe. PAL-003 began on January 5, 2010, and we report here data acquired through October 27, 2016.

\section{Study participants}

Individuals with PKU who completed a parent study with pegvaliase and were willing and able to maintain stable protein intake were eligible to enroll in the extension study. Sexually active participants were required to use two acceptable methods of contraception.

Patients were excluded if they were taking, other than pegvaliase, any medication to treat PKU or any injectable drugs containing PEG (e.g., medroxyprogesterone). Patients currently pregnant or planning to become pregnant (self or partner) or breastfeed during the study period were excluded. Patients who had a prior systemic hypersensitivity event (e.g., generalized hives, hypotension, anaphylaxis, or angioedema) to a PEG-containing product were excluded, with the exception that patients with a prior reaction to pegvaliase could be eligible for participation at the discretion of the principal investigator in consultation with the Sponsor's medical monitor. 


\section{Study drug administration}

Participants who enrolled in the extension study either continued their pegvaliase dosing regimen from their parent study or started at a higher dose, as determined by the primary investigator and medical monitor. The dose level and/or frequency were adjusted for each participant to achieve a plasma Phe concentration between 60 and $600 \mu \mathrm{mol} / \mathrm{L}$ or in response to an adverse event (AE).

Pegvaliase doses were similar to those previously tested in the parent studies [13, 14], between $2.5 \mathrm{mg} /$ week and $375 \mathrm{mg} /$ week or between $0.001 \mathrm{mg} / \mathrm{kg} /$ week and $5 \mathrm{mg} / \mathrm{kg} /$ week, administered up to 7 days/week; weight-based dosing allowed participants $>75 \mathrm{~kg}$ to receive a dose higher than $375 \mathrm{mg} /$ week, but a protocol change in October 2014 clarified an absolute maximum dose of $375 \mathrm{mg} /$ week.

The first dose of pegvaliase in the extension study was administered in the clinic. Injection sites were rotated between doses in common areas for subcutaneous injection (e.g., upper arm, thigh, or abdomen).

\section{Dietary intake}

Adherence to a Phe-restricted diet was not required, but participants were instructed to maintain a total protein intake consistent with their dietary intake at time of entry into the extension study. This was so that changes in endpoints would be attributable to the study drug rather than to changes in protein intake. When necessary in patients with low Phe levels, protein intake was increased under the supervision of the investigator. Average daily dietary protein and Phe intake was calculated using 3-day diet records before study visits.

\section{Hypersensitivity adverse events}

Participants were trained to recognize and respond to hypersensitivity AEs (HAEs) and were given epinephrine injectors for use in the event of an acute systemic hypersensitivity event, including potential anaphylaxis events. Investigators used clinical judgment to decide if a participant should receive premedication with a histamine receptor-1 antagonist, histamine receptor-2 antagonist, and/or antipyretic prior to subsequent pegvaliase dosing. In the event of an acute systemic hypersensitivity event, including potential anaphylaxis events, the dose of pegvaliase could be maintained, reduced, or interrupted at the discretion of the investigator. Starting in October 2014, in the event of acute systemic hypersensitivity that met Brown's severe criteria (i.e., hypoxia, hypotension, or neurologic compromise) [17], pegvaliase was withdrawn.

\section{Assessments}

Safety and plasma Phe concentration were assessed every 4 weeks and immune response every 12 weeks. A central laboratory was used to analyze plasma Phe concentrations.
Safety was assessed by vital signs, physical examination, AEs, and clinical laboratory tests (chemistry, hematology, and urinalysis). The incidence, exposure-adjusted event rate, and severity grade (per National Cancer Institute Common Terminology Criteria for Adverse Events [CTCAE], version 4.03 [18]: mild, moderate, severe, life-threatening, or death) of AEs were reported.

All AEs were coded according to Medical Dictionary for Regulatory Activities (MedDRA; version 18.0 [19]) preferred terms. AEs of special interest for purposes of additional safety monitoring included injection-site reactions, generalized skin reactions with duration $\geq 14$ days, arthralgia, HAEs, and acute systemic hypersensitivity events, which were identified using predefined search strategies. All potential acute systemic hypersensitivity events and AEs where epinephrine was administered were reviewed by an allergist/immunologist independent of the clinical site and Sponsor to identify acute systemic hypersensitivity events consistent with clinical anaphylaxis criteria as defined by National Institute of Allergy and Infectious Diseases/Food Allergy and Anaphylaxis Network (NIAID/FAAN) and Brown's severe criteria [17]. In addition to the Sponsor, an independent Data Monitoring Committee monitored the safety of participants, acting in an advisory capacity to the Sponsor.

Immunology testing was performed using validated assays to measure immunoglobulin G (IgG) and immunoglobulin M (IgM) responses to PAL, PEG, and neutralizing antibodies (NAbs). Serum samples for routine immunology testing were collected prior to dosing. In the event of an acute systemic hypersensitivity event, participants may have had an extra clinic visit scheduled, at the discretion of the investigator, at which samples were collected for anti-PAL and anti-pegvaliase immunoglobulin E (IgE) measurement.

\section{Statistical analysis}

Descriptive summaries of continuous variables (i.e., number of participants [n], mean, standard deviation [SD], median, and range) and of categorical variables (i.e., $\mathrm{n}$ and percentage) were analyzed by parent studies (all parent study data pooled together), the extension study, and overall (cumulative phase 2 treatment) to provide a robust population for evaluating trends in efficacy and safety outcomes with long-term treatment. Population mean data were summarized over time at intervals relative to baseline. The baseline value was defined as the last measurement before the first dose of pegvaliase (i.e., while patients were treatment naïve) in the parent study, unless indicated otherwise. The pegvaliase weekly dose was defined as the sum of the doses in the 7 days prior to each assessment. Daily dose categories were calculated using total drug amount $(\mathrm{mg})$ divided by entire duration (days) of study to allow comparison of dose between studies. 
The efficacy population included all participants who received at least one dose of pegvaliase and had at least one post-treatment plasma Phe concentration measurement. Plasma Phe measurements up to 14 days after a pegvaliase dose were included. Data presented as cumulative weeks of treatment include parent studies and the extension study.

The safety population included all participants who received at least one dose of pegvaliase [13, 14]. For AEs occurring more than once in a participant during the study, the AE of maximum severity was used in summaries. The duration of the parent studies was much shorter (up to 24 weeks of pegvaliase dosing) than the extension study (up to 102 months of pegvaliase dosing) $[13,14]$; therefore, event rates per person-years, adjusting for duration of exposure, are included for AE comparisons between parent and extension studies. Event rate comparisons between early $(<24$ weeks) and late $(>$ 24 weeks) treatment were made between different study populations (i.e., participants who discontinued the study during the first 24 weeks were not included in the late-treatment safety analysis).

The incidence rates of antibody positivity of PAL IgM, PAL IgG, PEG IgM, PEG IgG, and NAbs were summarized by treatment week. Antibody positivity, presented as percentage of participants, was calculated as the number of participants testing positive for an antibody divided by the total number of participants with data available at each timepoint. Plots were generated to explore the potential relationship between the incidence of antidrug antibodies and HAE frequency.

\section{Results}

\section{Participant disposition}

Sixteen investigators participated at 14 study centers in the United States. Eighty participants enrolled in one of the three parent studies, and 68 of these participants enrolled in the extension study. As of the cutoff date for data analysis, 37 participants remained in the extension study and 20 participants had discontinued the extension study ( $n=10$ due to withdrawal by participant decision, $n=5$ due to physician decision, $n=3$ lost to follow-up, and $n=2$ due to an $\mathrm{AE}$ ). Eleven participants completed the extension study and transferred into the phase 3 study PRISM-2.

\section{Participant baseline and demographic characteristics}

There were no meaningful differences in demographics or baseline characteristics between participants enrolled in the parent studies and the subset who continued into the extension study (Table 1 and Additional file 1: Table S1). Baseline mean (SD) protein intake was 69.4 (40.4) g/day. Baseline mean (SD) plasma Phe concentration for phase 2 participants was $1302.4(351.5) \mu \mathrm{mol} / \mathrm{L}$; in participants
Table 1 Participant demographic and baseline characteristics

\begin{tabular}{|c|c|}
\hline & Phase 2 participants $(N=80)$ \\
\hline \multicolumn{2}{|l|}{ Age at enrollment } \\
\hline Mean (SD), years & $28.3(8.8)$ \\
\hline Min, max, years & 16,56 \\
\hline$<18, \mathrm{n}(\%)$ & $3(3.8 \%)$ \\
\hline$\geq 18, \mathrm{n}(\%)$ & $77(96.3 \%)$ \\
\hline \multicolumn{2}{|l|}{ Sex } \\
\hline Female, $n(\%)$ & $46(57.5 \%)$ \\
\hline \multicolumn{2}{|l|}{ Race } \\
\hline White, $n(\%)$ & $78(97.5 \%)$ \\
\hline Weight & $n=79$ \\
\hline Mean (SD), kg & $80.4(24.5)$ \\
\hline Min, max, kg & $42.0,178.0$ \\
\hline Height & $n=74$ \\
\hline Mean (SD), cm & $167.2(9.6)$ \\
\hline Min, max, cm & $149.8,187.5$ \\
\hline Body mass index & $n=74$ \\
\hline Mean (SD), $\mathrm{kg} / \mathrm{m}^{2}$ & $28.5(7.7)$ \\
\hline Min, $\max , \mathrm{kg} / \mathrm{m}^{2}$ & $17.2,56.2$ \\
\hline$<25 \mathrm{~kg} / \mathrm{m}^{2}, n(\%)$ & $28(35.0 \%)$ \\
\hline 25 to $<30 \mathrm{~kg} / \mathrm{m}^{2}, n(\%)$ & $21(26.3 \%)$ \\
\hline$\geq 30 \mathrm{~kg} / \mathrm{m}^{2}, n(\%)$ & $25(31.3 \%)$ \\
\hline \multicolumn{2}{|l|}{ Plasma Phe } \\
\hline Mean (SD), $\mu \mathrm{mol} / \mathrm{L}$ & $1302.4(351.5)$ \\
\hline Min, $\max , \mu \mathrm{mol} / \mathrm{L}$ & $249.0,2214.0$ \\
\hline Protein intake $e^{a}$ & $n=36$ \\
\hline Mean (SD), g/day & $69.4(40.0)$ \\
\hline Min, max, g/day & $10.7,197.3$ \\
\hline Phe intake ${ }^{b}$ & $n=36$ \\
\hline Mean (SD), mg/day & 1975 (1583) \\
\hline Min, max, mg/day & 461,8419 \\
\hline
\end{tabular}

Baseline was defined as the last measurement before the first dose of pegvaliase (i.e., while patients were treatment naïve) in the parent study. All phase 2 data are included. Sample size is indicated if $N<80$ max maximum, min minimum, Phe phenylalanine, $S D$ standard deviation aprotein intake includes medical food and natural protein dietary intake and was calculated as the daily average intake over 3 days prior to the assessment point

${ }^{\mathrm{b}}$ Phe intake was calculated as the daily average intake over 3 days prior to the assessment point

who enrolled into the extension study, plasma Phe at entry to the extension study was 1022.4 (530.4) $\mu \mathrm{mol} / \mathrm{L}$.

\section{Pegvaliase exposure}

The mean dose of pegvaliase increased from 5.3 (6.8) $\mathrm{mg} /$ day in the parent studies to $26.2(17.9) \mathrm{mg} /$ day in the extension study (Additional file 1: Table S2). Overall mean treatment duration in phase 2 studies was 167.0 weeks (approximately 3.4 years), providing a total 


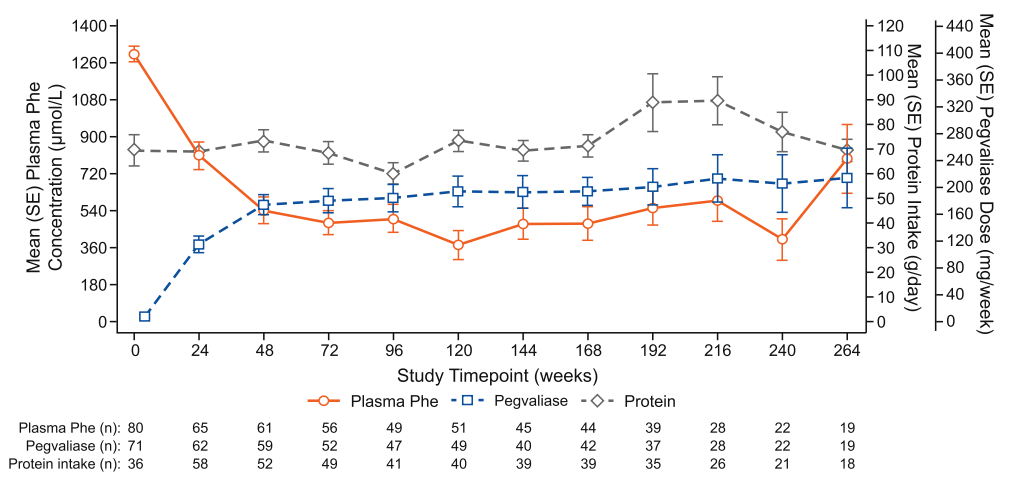

Fig. 1 Plasma Phe concentration, pegvaliase dose, and protein intake over time. Protein intake includes medical food and natural protein intake and was calculated as the daily average of intake over 3 days prior to the assessment point. Data are presented as mean (SE). All phase 2 data are included. Sample size reflects participants with data available at study timepoint; study is ongoing. Abbreviations: Phe phenylalanine, SE standard error

of 256 total patient-years of exposure (Table 2). Approximately $66.3 \%$ of participants (53 of 80 ) received pegvaliase for at least 2 years and $38.8 \%$ (31 of 80 ) received treatment for at least 4 years.

\section{Efficacy}

Mean (SD) plasma Phe decreased by $58.9 \%$ (39.0) from baseline to $541.6(515.5) \mu \mathrm{mol} / \mathrm{L}$ at Week 48 of treatment and by $72.3 \%(37.5)$ to $372.0(514.3) \mu \mathrm{mol} / \mathrm{L}$ at Week 120 (Additional file 1: Table S3).

A total of $78.7,80.0$, and $82.5 \%$ of participants achieved a plasma Phe concentration $\leq 120 \mu \mathrm{mol} / \mathrm{L}, \leq 360 \mu \mathrm{mol} / \mathrm{L}$, and $\leq 600 \mu \mathrm{mol} / \mathrm{L}$, respectively, at any time during the study (Table 3 ). Protein intake remained relatively constant during the trial (Fig. 1).

Mean plasma Phe concentration decreased as the mean pegvaliase dose was increased over time (Fig. 2). Both mean plasma Phe concentration and the mean weekly dose of pegvaliase remained relatively stable from Week 48 to Week 264 (almost 5 years) of pegvaliase treatment. Mean daily protein intake remained relatively stable throughout the study.

Table 2 Pegvaliase exposure

\begin{tabular}{ll}
\hline & Phase 2 participants $(N=80)$ \\
\hline Treatment duration, weeks & $167.0(111.1)$ \\
$\quad$ Mean (SD) & $0.71,370.4$ \\
$\quad$ Min, max & \\
Treatment duration, $n(\%)$ & $15(18.8 \%)$ \\
$<1$ year & $12(15.0 \%)$ \\
$\geq 1$ to $<2$ years & $10(12.5 \%)$ \\
$\geq 2$ to $<3$ years & $12(15.0 \%)$ \\
$\geq 3$ to $<4$ years & $31(38.8 \%)$ \\
$\geq 4$ years &
\end{tabular}

All data from phase 2 studies are included

max maximum, min minimum, $S D$ standard deviation

\section{Safety}

All participants reported at least one AE, with the most common AEs (by preferred term) being injection-site reaction $(72.5 \%$ of participants), injection-site erythema (67.5\%), headache (67.5\%), and arthralgia (65.0\%; Table 4). The majority of participants had AEs limited to mild $(12.5 \%)$ or moderate $(76.3 \%)$ severity; severe AEs were reported in $11.3 \%$ of participants. The overall $\mathrm{AE}$ event rate was 22.5 events per person-year; the event rate decreased during the extension study as compared to the parent studies (dropping from 58.3 to 18.6 events per person-year). There were no deaths (Table 5).

\section{Extension study}

Twenty-five participants (36.8\%) reported AEs that led to dose interruption or reduction; the most common AEs by preferred term were urticaria ( $n=5$ [7.4\% of participants]); arthralgia, generalized rash, hypersensitivity, and nausea $(n=3 \quad[4.4 \%$ of participants] each); and pruritus, injection-site rash, injection-site reaction, presyncope, diarrhea, viral gastroenteritis, gastrointestinal viral infection, nasopharyngitis, and viral infection $(n=2[2.9 \%$ of participants] each). The percentage of participants experiencing AEs was similar across dose groups $(93.2 \%$ with doses $<20 \mathrm{mg} /$ day; $83.6 \%$ with doses $\geq 20$ to $<40$; $86.3 \%$ with doses $\geq 40$ to $<60 ; 84.4 \%$ with doses $\geq 60 \mathrm{mg} /$ day).

Four participants (5.9\%) had AEs leading to early discontinuation of pegvaliase treatment; two of these participants also discontinued study participation due to AEs. One participant with two previous acute systemic hypersensitivity events of anaphlyaxis during parent study treatment had two additional events of anaphylaxis in the extension study and discontinued treatment due to AEs after the fourth event (acute systemic hypersensitivity events further described below); one participant discontinued treatment due to AEs of severe arthralgia and peripheral neuropathy; one participant withdrew consent and discontinued treatment after experiencing 
Table 3 Participants with plasma Phe $\leq 120 \mu \mathrm{mol} / \mathrm{L}$, $\leq 360 \mu \mathrm{mol} / \mathrm{L}$, or $\leq 600 \mu \mathrm{mol} / \mathrm{L}$

\begin{tabular}{lllll}
\hline & $n$ & \multicolumn{3}{l}{ Plasma Phe threshold, $\mathrm{n}(\%)$} \\
\cline { 3 - 5 } & & $\leq 120 \mu \mathrm{mol} / \mathrm{L}$ & $\leq 360 \mu \mathrm{mol} / \mathrm{L}$ & $\leq 600 \mu \mathrm{mol} / \mathrm{L}$ \\
\hline Overall $^{\text {a }}$ & 80 & $63(78.7 \%)$ & $64(80.0 \%)$ & $66(82.5 \%)$ \\
Extension study & & & & \\
Week 48 & 61 & $20(32.8 \%)$ & $28(45.9 \%)$ & $35(57.4 \%)$ \\
Week 96 & 49 & $17(34.7 \%)$ & $22(44.9 \%)$ & $30(61.2 \%)$ \\
Week 144 & 45 & $19(42.4 \%)$ & $25(55.6 \%)$ & $29(64.4 \%)$ \\
Week 192 & 39 & $13(33.3 \%)$ & $17(43.6 \%)$ & $23(58.9 \%)$ \\
Week 240 & 22 & $11(50.0 \%)$ & $13(59.1 \%)$ & $16(72.7 \%)$ \\
Week 264 & 19 & $5(26.3 \%)$ & $6(31.6 \%)$ & $8(42.1 \%)$ \\
Overall & 68 & $57(83.8 \%)$ & $58(85.2 \%)$ & $59(86.7 \%)$ \\
\hline
\end{tabular}

All data from phase 2 studies are included. Sample size reflects participants with data available at study timepoint; study is ongoing Phe phenylalanine

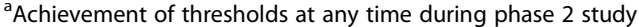

mild pruritus; and one participant with four cases of presyncope discontinued treatment after the fourth event. All AEs resolved.

Eleven (16.2\%) participants had a total of 16 serious AEs (SAEs) in the extension study, with two participants (2.9\%) reporting SAEs by preferred term of asthma; other SAEs were reported by one participant each: anaphylactic reaction (associated with a confirmed acute systemic hypersensitivity event described below), appendicitis, infectious diarrhea, hypotension, lymphadenopathy, staphylococcal infection, and suicide attempt; hypersensitivity and asthma in one participant; injection-related reaction, neutropenia, and bone marrow failure in one participant; and asthma, arthralgia, and peripheral neuropathy in one participant. All SAEs resolved.

The most common AEs by preferred term in the extension study were headache (63.2\%), nasopharyngitis $(58.8 \%)$, rash $(51.5 \%)$, injection-site reaction (51.5\%), arthralgia (50.0\%), and injection-site erythema (50.0\%). The most common AEs by event rate were urticaria (1.2 events/person-year) and injection-site bruising (1.0 events/person-year).

\section{Acute systemic hypersensitivity events}

One participant in the extension study experienced two acute systemic hypersensitivity events of anaphylaxis consistent with clinical NIAID/FAAN criteria as confirmed by an independent allergist/immunologist, both of which also met Brown's severe criteria. The coded AE preferred terms associated with these two events were hypersensitivity $(n=1)$ and anaphylactic reaction $(\mathrm{n}=1)$. Both cases resolved without sequelae, and the participant was not admitted to the hospital. The participant resumed pegvaliase dosing 3 days after the first acute systemic hypersensitivity event in the extension study and continued pegvaliase for 150 days, at which point the second event occurred and the participant discontinued pegvaliase. Drug-specific IgE was not detected at or near the time of the acute systemic hypersensitivity events.

\section{AEs of interest}

At least one HAE was reported by 62 of 68 participants (91.2\%) in the extension study. HAEs with the highest incidence in the extension study included rash (51.5\%),

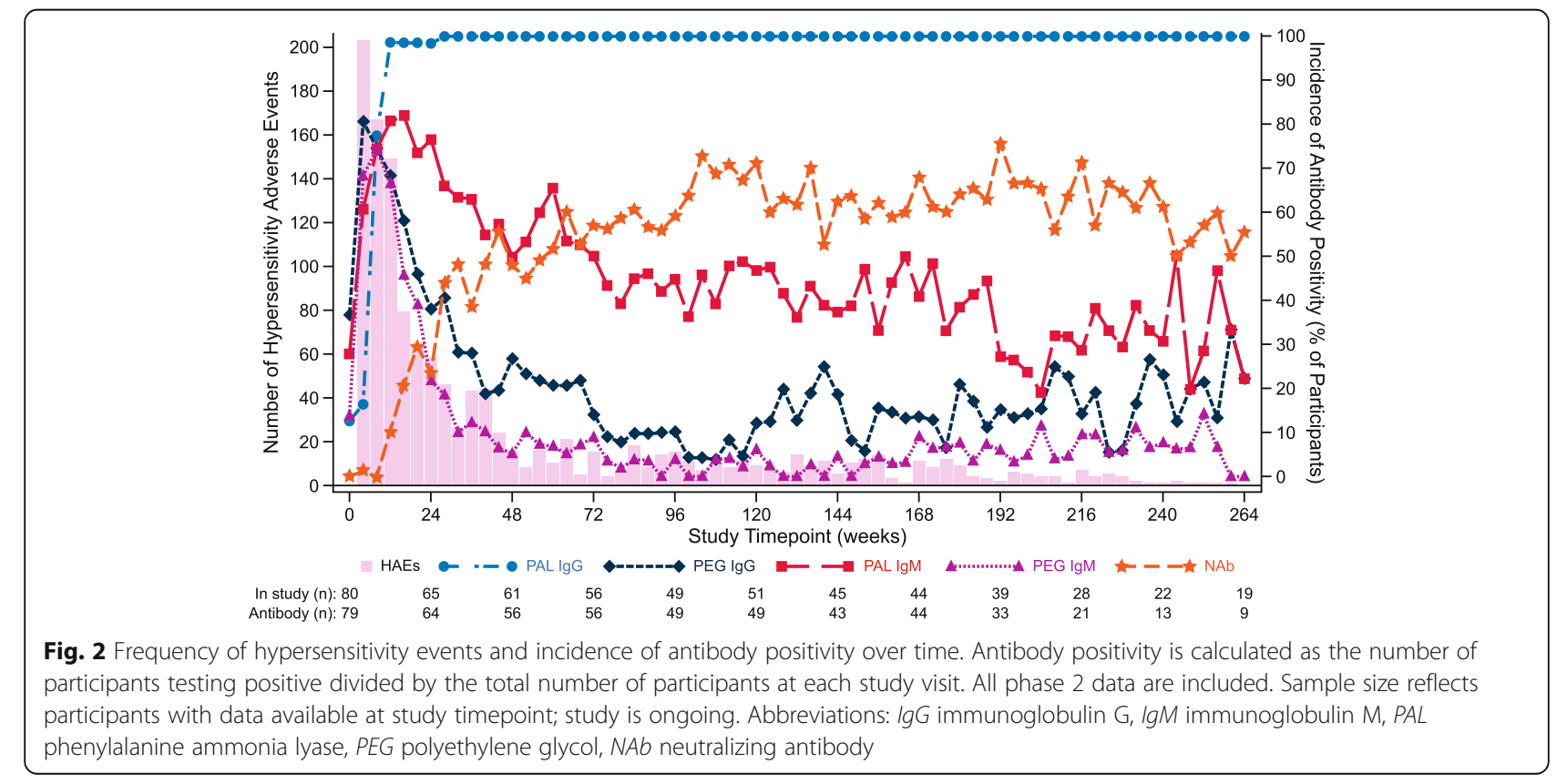


Table 4 AEs (incidence $\geq 25 \%$ all participants) reported as subject incidence (n, \%), and event rate (events/person-years) and total number of events

\begin{tabular}{|c|c|c|c|c|c|c|}
\hline \multirow[t]{3}{*}{ Exposure, person-years } & \multicolumn{2}{|c|}{ Parent study $(n=80)$} & \multicolumn{2}{|c|}{ Extension study $(n=68)$} & \multicolumn{2}{|c|}{ Overall phase 2 treatment $(n=80)^{a}$} \\
\hline & & 25.1 & & 230.0 & & 256.0 \\
\hline & $\begin{array}{l}\text { Incidence } \\
n(\%)\end{array}$ & $\begin{array}{l}\text { Event rate (total } \\
\text { number of events) }\end{array}$ & $\begin{array}{l}\text { Incidence } \\
n(\%)\end{array}$ & $\begin{array}{l}\text { Event rate (total } \\
\text { number of events) }\end{array}$ & $\begin{array}{l}\text { Incidence } \\
n(\%)\end{array}$ & $\begin{array}{l}\text { Event rate (total } \\
\text { number of events) }\end{array}$ \\
\hline AEs & 79 (98.8\%) & $58.3(1463)$ & $68(100 \%)$ & $18.6(4284)$ & $80(100 \%)$ & $22.5(5747)$ \\
\hline Headache & $33(41.3 \%)$ & $2.8(69)$ & $43(63.2 \%)$ & $0.97(223)$ & $54(67.5 \%)$ & $1.1(292)$ \\
\hline Nasopharyngitis & $15(18.8 \%)$ & $0.6(15)$ & $40(58.8 \%)$ & $0.43(98)$ & $47(58.8 \%)$ & $0.44(113)$ \\
\hline Rash & $22(27.5 \%)$ & $1.3(32)$ & $35(51.5 \%)$ & $0.51(118)$ & $46(57.5 \%)$ & $0.59(150)$ \\
\hline Injection-site reaction & $45(56.3 \%)$ & $9.1(229)$ & $35(51.5 \%)$ & $0.50(115)$ & $58(72.5 \%)$ & $1.3(344)$ \\
\hline Arthralgia & $41(51.3 \%)$ & $5.6(140)$ & $34(50.0 \%)$ & $0.74(171)$ & $52(65.0 \%)$ & $1.2(311)$ \\
\hline Injection-site erythema & $36(45.0 \%)$ & $3.6(91)$ & $34(50.0 \%)$ & $0.68(157)$ & $54(67.5 \%)$ & $0.97(248)$ \\
\hline Injection-site bruising & $21(26.3 \%)$ & $1.2(30)$ & $33(48.5 \%)$ & $1.0(240)$ & $47(58.8 \%)$ & $1.1(270)$ \\
\hline Upper respiratory tract infection & $17(21.3 \%)$ & $0.72(18)$ & $31(45.6 \%)$ & $0.64(147)$ & 39 (48.8\%) & $0.65(165)$ \\
\hline Oropharyngeal pain & $11(13.8 \%)$ & $0.48(12)$ & $31(45.6 \%)$ & $0.28(64)$ & $37(46.3 \%)$ & $0.30(76)$ \\
\hline Urticaria & $8(10.0 \%)$ & $0.44(11)$ & $30(44.1 \%)$ & $1.2(281)$ & $33(41.3 \%)$ & $1.1(292)$ \\
\hline Nausea & $24(30.0 \%)$ & $1.3(33)$ & $30(44.1 \%)$ & $0.27(62)$ & $41(51.3 \%)$ & $0.37(95)$ \\
\hline Cough & $15(18.8 \%)$ & $0.68(17)$ & $30(44.1 \%)$ & $0.20(45)$ & $39(48.8 \%)$ & $0.24(62)$ \\
\hline Back pain & $5(6.3 \%)$ & $0.40(10)$ & $29(42.6 \%)$ & $0.30(70)$ & $32(40.0 \%)$ & $0.31(80)$ \\
\hline Pruritus & $15(18.8 \%)$ & $0.92(23)$ & 27 (39.7\%) & $0.29(66)$ & $33(41.3 \%)$ & $0.35(89)$ \\
\hline Diarrhea & $12(15.0 \%)$ & $0.68(17)$ & $25(36.8 \%)$ & $0.30(70)$ & $31(38.8 \%)$ & $0.34(87)$ \\
\hline Vomiting & $9(11.3 \%)$ & $0.76(19)$ & $24(35.3 \%)$ & $0.22(51)$ & $28(35.0 \%)$ & $0.27(70)$ \\
\hline Contusion & $5(6.3 \%)$ & $0.20(5)$ & $23(33.8 \%)$ & $0.24(56)$ & $26(32.5 \%)$ & $0.24(61)$ \\
\hline Pain in extremity & $14(17.5 \%)$ & $0.76(19)$ & $23(33.8 \%)$ & $0.23(52)$ & $30(37.5 \%)$ & $0.28(71)$ \\
\hline Pyrexia & $15(18.8 \%)$ & $0.76(19)$ & $23(33.8 \%)$ & $0.15(35)$ & $33(41.3 \%)$ & $0.21(54)$ \\
\hline Injection-site pruritus & $9(11.3 \%)$ & $0.60(15)$ & $21(30.9 \%)$ & $0.32(73)$ & $25(31.3 \%)$ & $0.34(88)$ \\
\hline Myalgia & $9(11.3 \%)$ & $0.44(11)$ & 21 (30.9\%) & $0.28(64)$ & $26(32.5 \%)$ & $0.29(75)$ \\
\hline Sinusitis & $5(6.3 \%)$ & $0.20(5)$ & $21(30.9 \%)$ & $0.14(32)$ & $24(30.0 \%)$ & $0.15(37)$ \\
\hline Dizziness & $20(25.0 \%)$ & $1.2(30)$ & $20(29.4 \%)$ & $0.22(51)$ & $36(45.0 \%)$ & $0.32(81)$ \\
\hline Abdominal pain & $8(10.0 \%)$ & $0.44(11)$ & $18(26.5 \%)$ & $0.16(36)$ & $22(27.5 \%)$ & $0.18(47)$ \\
\hline
\end{tabular}

Event rate was calculated as total number of events divided by person-years of exposure. Incidence rates counted participants who reported more than 1 adverse event within a preferred term only once

$A E$ adverse event

${ }^{\text {a }}$ All phase 2 data are included

arthralgia (50.0\%), urticaria (44.1\%), pruritus (39.7\%), and pyrexia (33.8\% [Tables 4 and 5]).

Severe HAEs occurring in the extension study included arthralgia $(n=2)$, asthma $(\mathrm{n}=1)$, and anaphylactic reaction $(\mathrm{n}=1$, associated with a confirmed acute systemic hypersensitivity event described above). The frequency of HAEs decreased in the extension study compared to the parent studies, with an exposure-adjusted HAE rate of 14.9 events/person-year in the parent studies and 4.3 events/person-year in the extension study. The frequency of injection-site reactions, generalized skin reaction lasting $\geq 14$ days, injection-site skin reaction lasting $\geq 14$ days, and arthralgia also decreased in the extension study compared to the parent studies.
No events of serum sickness were reported during the extension study. No events suggesting immune complex-mediated end-organ damage, including renal failure, hemolytic anemia, serositis (such as peritonitis, pericarditis, and pleuritis), central nervous system manifestations (such as cerebrovascular accidents or transient ischemic attacks), or myocardial ischemic events related to pegvaliase were observed.

\section{Immunogenicity}

All participants developed a PAL IgG response in the parent studies, and the incidence rate was sustained through the long-term extension study. The majority of participants developed a PAL IgM response, which decreased over time, with incidence rates of $76.6 \%$ at Week 
Table 5 AEs reported by subject incidence (n, \%), event rate (events/person-years), and total number of events

\begin{tabular}{|c|c|c|c|c|c|c|}
\hline \multirow[t]{3}{*}{ Exposure, person-years } & \multicolumn{2}{|c|}{ Parent study $(n=80)$} & \multicolumn{2}{|c|}{ Extension study $(n=68)$} & \multicolumn{2}{|c|}{$\begin{array}{l}\text { Overall phase } 2 \\
\text { treatment }(n=80)^{\mathrm{a}}\end{array}$} \\
\hline & & 25.1 & & 230.0 & & 256.0 \\
\hline & $\begin{array}{l}\text { Incidence } \\
n(\%)\end{array}$ & $\begin{array}{l}\text { Event rate (total } \\
\text { number of events) }\end{array}$ & $\begin{array}{l}\text { Incidence } \\
n(\%)\end{array}$ & $\begin{array}{l}\text { Event rate (total } \\
\text { number of events) }\end{array}$ & $\begin{array}{l}\text { Incidence } \\
n(\%)\end{array}$ & $\begin{array}{l}\text { Event rate (total } \\
\text { number of events) }\end{array}$ \\
\hline$\overline{\mathrm{AES}}$ & 79 (98.8\%) & $58.3(1463)$ & $68(100 \%)$ & $18.6(4284)$ & $80(100 \%)$ & $22.5(5747)$ \\
\hline AEs causing early pegvaliase discontinuation & $5(6.3 \%)$ & $0.20(5)$ & $4(5.9 \%)$ & $0.05(12)$ & $9(11.3 \%)$ & $0.07(17)$ \\
\hline SAES & $4(5.0 \%)$ & $0.16(4)$ & $11(16.2 \%)$ & $0.07(16)$ & $15(18.8 \%)$ & $0.08(20)$ \\
\hline SAEs causing early pegvaliase discontinuation & $1(1.3 \%)$ & $0.04(1)$ & $2(2.9 \%)$ & $0.01(3)$ & $3(3.8 \%)$ & $0.02(4)$ \\
\hline Death & 0 & 0 & 0 & 0 & 0 & 0 \\
\hline \multicolumn{7}{|l|}{ AEs of special interest } \\
\hline Hypersensitivity events & $69(86.3 \%)$ & $14.9(373)$ & $62(91.2 \%)$ & $4.3(997)$ & $75(93.8 \%)$ & $5.4(1370)$ \\
\hline Generalized skin reaction ( $\geq 14$ days) & $9(11.3 \%)$ & $0.44(11)$ & $37(54.4 \%)$ & $0.40(93)$ & $39(48.8 \%)$ & $0.41(104)$ \\
\hline Injection-site reaction & $68(85.0 \%)$ & $19.1(479)$ & $59(86.8 \%)$ & $3.8(875)$ & $76(95.0 \%)$ & $5.3(1354)$ \\
\hline Injection-site skin reaction ( $\geq 14$ days) & $13(16.3 \%)$ & $0.88(22)$ & $25(36.8 \%)$ & $0.27(62)$ & $36(45.0 \%)$ & $0.33(84)$ \\
\hline Arthralgia & $41(51.3 \%)$ & $5.6(140)$ & $34(50.0 \%)$ & $0.74(171)$ & $52(65.0 \%)$ & $1.2(311)$ \\
\hline $\begin{array}{l}\text { Acute systemic hypersensitivity event of } \\
\text { anaphylaxis }\end{array}$ & $3(3.8 \%)$ & $15.9(4)$ & $1(1.5 \%)$ & $0.01(2)$ & $3(3.8 \%)$ & $0.02(6)$ \\
\hline $\begin{array}{l}\text { Acute systemic hypersensitivity event per Brown's } \\
\text { severe criteria }\end{array}$ & $1(1.3 \%)$ & $0.04(1)$ & $1(1.5 \%)$ & $0.01(2)$ & $2(2.5 \%)$ & $0.01(3)$ \\
\hline
\end{tabular}

Event rate was calculated as total number of events divided by person-years of exposure

$A E$ adverse event, $S A E$ serious adverse event

${ }^{\text {a }}$ All phase 2 data are included

24, 50.0\% at Week 48 , and $46.9 \%$ at Week 120 . Participants developed a transient anti-PEG response, with the incidence of PEG IgM and PEG IgG peaking at Week 8 and then decreasing to baseline levels by Week 36 of pegvaliase treatment. The incidence rate of NAb increased from baseline, with the majority of participants developing a NAb response that was sustained during long-term treatment; $23.4 \%$ of participants were positive at Week $24,48.2 \%$ at Week 48 , and $71.4 \%$ at Week 120 of treatment.

The frequency of HAEs was highest during early treatment when the incidence rates of PAL IgM, PEG IgM, and PEG IgG antibodies were highest (Fig. 2). The number of HAEs per study week decreased over time, as incidence rates of PEG IgM, PEG IgG, and PAL IgM antibodies decreased, and incidence rates of PAL IgG and NAb antibodies were sustained.

\section{Discussion}

The results from this phase 2 extension study indicate that pegvaliase produced a meaningful and sustained reduction in mean plasma Phe concentration in adults with PKU. The mean decrease in plasma Phe levels to $<600 \mu \mathrm{mol} / \mathrm{L}$ was sustained through 264 weeks (approximately 5 years) of treatment with 256 total patient-years of exposure.

At Week 48 of treatment, almost half (45.9\%) of participants had achieved a plasma Phe concentration of $\leq 360 \mu \mathrm{mol} / \mathrm{L}$ (the recommended upper limit in the United States established by ACMG), and a majority (57.4\%) of participants achieved a plasma Phe concentration of $\leq 600 \mu \mathrm{mol} / \mathrm{L}$ (the recommended upper limit established by the European Society for Phenylketonuria and Allied Disorders for adults) at least once during the extension study [2, 9]. These proportions of participants remained similar throughout most assessment points during long-term pegvaliase treatment.

Total protein intake at baseline (mean $69.4 \mathrm{~g} /$ day) reported by study participants was substantially higher than the $6 \mathrm{~g} /$ day of natural protein consumed by PKU patients with severe disease. Most study participants were not following a Phe-restricted diet and had protein intake similar to the intake recommended for the unaffected population in the US (about $56 \mathrm{~g} /$ day protein for a $70-\mathrm{kg}$ adult) [16, $20]$. With pegvaliase treatment, this study population was able to experience sustained plasma Phe reductions with relatively high protein intake at baseline and throughout the study, particularly during Weeks 168-216.

There were no new safety signals detected during the extension study; the AEs that occurred in the extension study were similar to the AEs reported in the parent studies, including those that led to participants discontinuing pegvaliase early (i.e., acute systemic hypersensitivity event, pruritus, and presyncope in one subject each, and arthralgia and peripheral neuropathy in one subject) in the extension study. Event rates for AEs were higher during 
early treatment in parent studies, when participants were initiating treatment and increasing the dosage of pegvaliase. The frequency of AEs decreased in participants continuing long-term treatment, when the maintenance dose of pegvaliase was achieved. Most participants had AEs limited to mild or moderate severity, all of which resolved.

The safety and immunogenicity results presented here were consistent with previous pegvaliase study reports $[13,14,21]$. As pegvaliase is a bacterial protein, HAEs due to an immune response were expected [20, 22]. The frequency of HAEs was highest during early treatment in the parent studies, when the incidence of PAL IgM, PEG IgM, and PEG IgG antibodies were highest. Overall, the number of HAEs per study week decreased over time as the incidence of PAL IgM, PEG IgM, and PEG IgG antibodies decreased and the incidence of PAL IgG and NAb antibodies increased. The initial antibody response, comprised heavily of pentameric IgM (PEG IgM and PAL IgM), appears to be more efficient at complement activation than the PAL IgG-dominant antibody response that occurs over time [20, 23, 24]. Anti-PEG antibodies are thought to bind to unobscured PEG epitopes on the surface of pegvaliase and activate complement by bringing multiple receptors of the fragment crystallizable $(\mathrm{Fc})$ region of the antibody in close proximity to each other [20,24]. Together, these observations, in conjunction with the lack of drug-specific IgE positivity, suggest that the likely mechanism of hypersensitivity reactions during therapy with pegvaliase is type III immune complex-mediated hypersensitivity [20, 24].

The magnitude of immune response exhibited by an individual influences the dosage needed to achieve blood Phe reductions, likely due to immune-mediated clearance. A wide range of pegvaliase doses were used for plasma Phe reductions in the extension study, and all participants developed an antibody response to pegvaliase. Subjects with lower antibody responses experienced greater blood Phe reductions in early treatment. As the immune response matures and the pegvaliase dose increases, subjects with a more robust antibody response also experience plasma Phe reductions [10, 16, 25, 26].

The design of this study was open-label, with no placebo or comparator group. Participants were directed to maintain a stable diet monitored by diet diary entries. However, due to the long duration of the study, some variations in diet could have contributed to variability in plasma Phe, and data were not collected to quantify changes in protein intake from medical food versus natural protein. Variability in the Phe levels at later study timepoints likely results from a smaller available sample size due to withdrawals and to participants not yet reaching timepoints as of data cutoff date. A limitation in the descriptive summary for the phase 2 studies is that individual participant data were not evaluated to assess fluctuations or persistency of results. It will be important to assess these in the future, particularly plasma Phe efficacy responses. Individuals with fluctuations in plasma Phe may be difficult to detect, as mean population analyses appear relatively stable over time. In addition, efficacy analyses of achievement of plasma Phe thresholds included participants who reached a specified threshold at least once during the study and did not measure persistency of that achievement.

Many participants continued pegvaliase treatment for an extended period of time. As of October 2016, 66 and 39\% of participants who enrolled in the extension study had reached $\geq 2$ years and $\geq 4$ years of pegvaliase treatment, respectively. Currently, about half of the participants remain in the extension study, 20 participants discontinued (primarily due to participant or physician decision), and 11 participants completed the study and subsequently enrolled in a phase 3 pegvaliase study. Phase 3 clinical studies have examined the safety and efficacy of pegvaliase in a randomized withdrawal trial [15] and a long-term extension study [16] with plasma Phe, neuropsychiatric, dietary protein intake, and safety endpoints [21].

\section{Conclusions}

Pegvaliase may address an unmet need for many individuals with PKU who have difficulty controlling blood Phe concentration. The PAL-003 phase 2 extension study demonstrated substantial efficacy of pegvaliase in maintaining reduced blood Phe concentration with long-term treatment (up to 5 years). Among participants able to continue with long-term treatment, many reached plasma Phe concentrations within guideline-recommended levels. Overall, the safety profile was managable for the majority of subjects, with AEs limited to mild or moderate severity and $\mathrm{AE}$ rates decreasing in participants receiving long-term pegvaliase treatment.

\section{Appendix \\ PAL-003 investigators and study sites}

D. Adams, Albany Medical College; B.K. Burton, Ann \& Robert H. Lurie Children's Hospital of Chicago; D. Dimmock, Medical College of Wisconsin; K. Goodin, Weisskopf Child Evaluation Center; D. Grange, St. Louis Children's Hospital; C.O. Harding, Oregon Health \& Science University; R. Hillman, University of Missouri; H. Levy, Boston Children's Hospital; N. Longo, University of Utah; W. Rizzo, University of Nebraska Medical Center; N. Shur, Albany Medical College; J.A. Thomas, Children's Hospital of Colorado; J. Vockley, Children's Hospital of Pittsburgh; M.P. Wasserstein, Icahn School of Medicine at Mount Sinai; R. Zori, University of Florida. 


\section{Additional file}

Additional file 1: Table S1. Participant demographic and baseline characteristics in extension study. Table S2. Pegvaliase exposure. Table S3. Blood Phe concentration by study week. (DOCX 33 kb)

\begin{abstract}
Abbreviations
ACMG: American College of Medical Genetics and Genomics; AE: Adverse event; CTCAE: Common Terminology Criteria for Adverse Events; HAE: Hypersensitivity adverse event; IgE: Immunoglobulin E; IgG: Immunoglobulin G; IgM: Immunoglobulin M; MedDRA: Medical Dictionary for Regulatory Activities; NAb: Neutralizing antibody; NIAID/ FAAN: National Institute of Allergy and Infectious Diseases/Food Allergy and Anaphylaxis Network; PEG: Polyethylene glycol; Phe: Phenylalanine; PKU: Phenylketonuria; SAE: Serious adverse event; SD: Standard deviation; SMQ: Standardized MedDRA query
\end{abstract}

\section{Acknowledgements}

The authors would like to thank the phase 2 study investigators, study coordinators, and study site support staff; Dr. Agnella Izzo Matic, representing MedAccess, for medical writing and editorial assistance; Dr. Vanessa Birardi of BioMarin Pharmaceutical Inc. for critical review of the manuscript; Ben Wang of BioMarin Pharmaceutical Inc. for statistical support; and Dr. Suyash Prasad and Dr. Saba Sile for overseeing portions of the study and contributing to the design.

\section{Funding}

BioMarin Pharmaceutical Inc. provided funding for the study and for manuscript preparation.

\section{Availability of data and materials}

The data that support the findings of this study are available from BioMarin Pharmaceutical Inc., but restrictions apply to the availability of these data and so they are not publicly available. Data are available from the authors upon reasonable request and with permission of BioMarin Pharmaceutical Inc.

\section{Authors' contributions}

CD was the medical monitor. ML performed the statistical analysis. All authors interpreted the results, reviewed and contributed to the manuscript, and were fully responsible for the content and editorial decisions related to this manuscript. All authors read and approved the final manuscript.

\section{Ethics approval and consent to participate}

All individuals consented to participate in the study, which was conducted in accordance with the Declaration of Helsinki of 1975, as revised in 2008.

\section{Consent for publication}

Not applicable.

\section{Competing interests}

$J V, B K B, R Z, J A T, M P W$, and NL are investigators of BioMarin Pharmaceutical Inc. clinical trials, and RZ, JAT, and NL are on the steering committee for the pegvaliase clinical program. NL reports grants (Aeglea, BioMarin, Genzyme, Horizon, Lumos Pharma, Protalix, Retrophin, Shire, Stealth Therapeutics, Ultragenix), consultant fees (Aeglea, BioMarin, Censa Pharmaceuticals, Dimension Therapeutics, Genzyme, Hemoshear, Horizon, Lumos Pharma, Moderna, Mitobridge, Pfizer, Retrophin, Stealth Therapeutics), participation in clinical trials (Aeglea, BioMarin, Genzyme, Horizon, Protalix, Retrophin, Shire, Stealth Therapeutics, Ultragenix), and travel fees (BioMarin, Cello Health Sciences, Lumos Pharma, SigmaTau/AlphaSigma). JV has received research support (BioMarin). BKB has participated in advisory boards (BioMarin, ReGenXBio), received research support (Shire), received consulting fees (Alexion, Shire, BioMarin), and participated as trial investigator (BioMarin, Shire, Ultragenyx, Alexion Armagen, Cytonet). JAT has participated in advisory boards and received research support (BioMarin). CD, ML, KL, JJ, and $\mathrm{KL}$ are BioMarin Pharmaceutical Inc. employees and stockholders.

\section{Publisher's Note}

Springer Nature remains neutral with regard to jurisdictional claims in published maps and institutional affiliations.

\section{Author details}

${ }^{1}$ Department of Pediatrics, Division of Medical Genetics, University of Utah, 295 Chipeta Way, Salt Lake City, UT 84108, USA. ²Division of Genetics and Metabolism, University of Florida, PO Box 100296 UFHSC, Gainesville, FL 32610, USA. ${ }^{3}$ Department of Pediatrics, The Children's Hospital at Montefiore, 3415 Bainbridge Ave, Bronx, NY 10467, USA. ${ }^{4}$ Department of Pediatrics, Division of Medical Genetics, University of Pittsburgh and Children's Hospital of Pittsburgh, 4401 Penn Avenue, Pittsburgh, PA 15224, USA. ${ }^{5}$ Department of Pediatrics, Division of Genetics, Birth Defects \& Metabolism, Ann \& Robert H. Lurie Children's Hospital of Chicago, 225 E. Chicago Ave, Chicago, IL 60611, USA. ${ }^{6}$ BioMarin Pharmaceutical Inc, 105 Digital Drive, Novato, CA 94949, USA. ${ }^{7}$ Department of Pediatrics, Section of Clinical Genetics and Metabolism, University of Colorado Hospital, 12605 E. 16th St, Aurora, CO 80045, USA.

Received: 1 February 2018 Accepted: 27 June 2018

Published online: 04 July 2018

\section{References}

1. Therrell BL Jr, Lloyd-Puryear MA, Camp KM, Mann MY. Inborn errors of metabolism identified via newborn screening: ten-year incidence data and costs of nutritional interventions for research agenda planning. Mol Genet Metab. 2014:113:14-26.

2. Vockley J, Andersson HC, Antshel KM, et al. Phenylalanine hydroxylase deficiency: diagnosis and management guideline. Genet Med. 2014;16:188-200.

3. Regier DS, Greene CL. Phenylalanine hydroxylase deficiency. In: Pagon RA, Adam MP, Ardinger HH, editors. GeneReviews [Internet]. Seattle: University of Washington; 2017.

4. Jurecki ER, Cederbaum S, Kopesky J, et al. Adherence to clinic recommendations among patients with phenylketonuria in the United States. Mol Genet Metab. 2017;120:190-7.

5. MacDonald A, Gokmen-Ozel H, van Rijn M, Burgard P. The reality of dietary compliance in the management of phenylketonuria. J Inherit Metab Dis. 2010;33:665-70

6. Burton BK, Grange DK, Milanowski A, et al. The response of patients with phenylketonuria and elevated serum phenylalanine to treatment with oral sapropterin dihydrochloride (6R-tetrahydrobiopterin): a phase II, multicentre, open-label, screening study. J Inherit Metab Dis. 2007:30:700-7.

7. Brown CS, Lichter-Konecki U. Phenylketonuria (PKU): a problem solved? Mol Genet Metab Rep. 2016:6:8-12.

8. Kuvan [package insert]. BioMarin Pharmaceutical, Inc,: Novato;2016.

9. van Spronsen FJ, van Wegberg AM, Ahring K, et al. Key European guidelines for the diagnosis and management of patients with phenylketonuria. Lancet Diabetes Endocrinol. 2017:5:743-56.

10. Palynziq [package insert]. Novato: BioMarin Pharmaceutical Inc:; 2018.

11. Longo N, Harding CO, Burton BK, et al. Single-dose, subcutaneous recombinant phenylalanine ammonia lyase conjugated with polyethylene glycol in adult patients with phenylketonuria: an open-label, multicentre, phase 1 dose-escalation trial. Lancet. 2014;384:37-44.

12. Bell SM, Wendt DJ, Zhang Y, et al. Formulation and PEGylation optimization of the therapeutic PEGylated phenylalanine ammonia lyase for the treatment of phenylketonuria. PLoS One. 2017;12:e0173269.

13. Thomas JA, Longo $\mathrm{N}$, Zori $\mathrm{R}$, et al. Evaluation of multiple dosing regimens in phase 2 studies of rAvPAL-PEG (BMN 165) for control of blood phenylalanine levels in adults with phenylketonuria. Mol Genet Metab. 2015;114:364-5.

14. Zori R, Thomas JA, Shur N, et al. Evaluation of an induction, titration, and maintenance dosing regimen in a phase 2 study of RAVPAL-PEG (BMN 165) for control of blood phenylalanine levels in adults with phenylketonuria (PKU). Mol Genet Metab. 2015:114:368-9.

15. Harding CO, Amato RS, Stuy M, et al. Pegvaliase for the treatment of phenylketonuria: a pivotal, double-blind randomized discontinuation phase 3 clinical trial. Mol Genet Metab. 2018;124:20-6.

16. Thomas J, Levy H, Amato S, et al. Pegvaliase for the treatment of phenylketonuria: results of a long-term phase 3 clinical trial program (PRISM). Mol Genet Metab. 2018;124:27-38.

17. Brown SGA. Clinical features and severity grading of anaphylaxis. J Allergy Clin Immunol. 2004;114:371-6. 
18. National Cancer Institute. Common terminology criteria for adverse events, v4.03: NIH publication \#09-7473; 2010. http://www.hrc.govt.nz/sites/default/ files/CTCAE\%20manual\%20-\%20DMCC.pdf.

19. Hershfield MS, Ganson NJ, Kelly SJ, Scarlett EL, Jaggers DA, Sundy JS. Induced and pre-existing anti-polyethylene glycol antibody in a trial of every 3-week dosing of pegloticase for refractory gout, including in organ transplant recipients. Arthritis Res Ther. 2014;16:R63.

20. Krishna M, Nadler SG. Immunogenicity to biotherapeutics - the role of antidrug immune complexes. Front Immunol. 2016;7:21.

21. Harding CO, Amato S, Vockley J, et al. Phase 3 PRISM-1 and PRISM-2 clinical trial results: to evaluate the efficacy and safety of pegvaliase for the treatment of adults with phenylketonuria (PKU). J Inherit Metab Dis. 2016; 39(Suppl 1):S107-8.

22. Chaplin DD. Overview of the immune response. J Allergy Clin Immunol. 2010;125:S3-S23.

23. Dunkelberger JR, Song W-C. Complement and its role in innate and adaptive immune responses. Cell Res. 2010;20:34-50.

24. Verhoef JJF, Carpenter JF, Anchordoquy TJ, Schellekens H. Potential induction of anti-PEG antibodies and complement activation toward PEGylated therapeutics. Drug Discov Today. 2014;19:1945-52.

25. Lau K, Gupta S, Olbertz J, et al. Phase 3 PRISM clinical trials: evaluating circulating immune complexes and clinical impact after pegvaliase treatment of adults with phenylketonuria. American College of Medical Genetics Annual Meeting 2018: Abstract 262. https://acmg.expoplanner. com/index.cfm?do=expomap.sess\&event_id=12\&session_id=7098.

26. Gupta S, Lau K, Olbertz J, et al. Maturation of immune response against pegvaliase is associated with reduced hypersensitivity and improved efficacy in the phase 3 clinical trials. Molecular Genetics and Metabolism. 2018;123:236-7. (Abstract 54)

\section{Ready to submit your research? Choose BMC and benefit from:}

- fast, convenient online submission

- thorough peer review by experienced researchers in your field

- rapid publication on acceptance

- support for research data, including large and complex data types

- gold Open Access which fosters wider collaboration and increased citations

- maximum visibility for your research: over $100 \mathrm{M}$ website views per year

At BMC, research is always in progress.

Learn more biomedcentral.com/submissions 